\title{
Blast impact on structures of underground parking
}

\author{
P. P. Procházka ${ }^{1}$, A. N. Kravtsov ${ }^{2} \&$ S. Peskova ${ }^{1}$ \\ ${ }^{1}$ Czech Association of Civil Engineers, Prague, Czech Republic \\ ${ }^{2}$ Czech Technical University in Prague, Civil Engineering, \\ Structural Mechanics, Czech Republic
}

\begin{abstract}
In big cities underground spaces are built up for subways, underground parking and tunnels, etc. These rooms are threatened by terrorist attacks and not only human lives can be lost but also extensive material damage can be expected. This is why it is of great importance to predict dynamic impacts of explosives, which can then be transformed to static statistically evaluated loading. In this paper the impact of explosion and air strike wave is formulated and solved. Gas dynamics and dynamic response of soil with process of dissipation of air-strike energy are considered. This means that some part of this energy is transferred into structures and soil mass. It appears that for contact explosions on the soil surface this part can be up to $30 \%$ of the total explosion energy (in soft soils). The variables to be calculated are mass density of gas, the velocity of movements and the internal energy. The latter covers the influence of the gas pressure, being given for the adiabatic state. The air is linearly related to the internal energy of a unit mass of the gas, and the density, while in the neighborhood of the source of explosion the pressure changes nonlinearly with respect to the gas density. Time dependent finite element solution is compared with results published in Lucy, L.B. (1977). A numerical approach to testing of the fission hypothesis. Astron. J. 82, 1013.
\end{abstract}

Keywords: underground parking, striking wave due to explosion, time development of gas pressure, impact load.

\section{Introduction}

Numerical simulation of gas explosion is very particular as the system of equations describing the process are nonlinear and of the first order. Moreover, 
movement of interfacial boundary between subdomain simulating neighborhood of explosion and the virgin air (gas) moves according to the current situation. Consequently, large movements are expected. In order to describe such displacements of the gas couple of numerical approaches exists. One of the most suiting appears smooth hydrodynamics particle method (SHP), which was historically developed for astrophysical purposes, [1, 2]. The inherent benefit of the SHP formulation consists in transformation of partial differential equations to a system of linear algebraic using regularization. This transformation is, among others, suitable for parallel computations. Recently, SHP has grown into a successful and respected numerical tool. In particular, this method does not differ between 3D, 2D and 1D problems, as the problems defined in higher order spaces can be simulated as easy as that in $1 \mathrm{D}$. An excellent review of the advantages and recent progress in SHP can be found in [3, 4]. Some problems occur when geometrical boundary conditions should be involved. Authors of [5] proposed the ghost particle method, in which some particles are located outside the domain. Heat conduction problem is solved in [6], where Taylor series expansion approximates the regularization kernels.

This paper partly starts with ideas of Veselovsky and Kurepin [7], where the problem of explosion in underground parking is solved. More detailed analysis is submitted in [8], where two-dimensional problems of gas dynamics are comprehensively discussed. The general approach for hydrodynamic processes involving strike waves and high temperature can be found in [9]. This information is collected into a formulation of loading acting against fixed walls of underground parking. The solution of the problem is done in terms of SHP.

\section{Methodology of load calculation}

The general problem of definition of loading on structures due to explosion and air strike waves is a complicated topic of solid mechanics. It covers a combined solution of gas-dynamics, dynamics of soil and building structures involving processes of dissipation of air-strike energy. That means that some part of this energy is transferred to structures and soil massive. For contact explosions on soil surfaces this part can be up to $30 \%$ of explosion energy (on soft soils). But in this case with lifted charge (center of explosion) the problem can be formulated in a simpler way.

Definition of the load will be split into two steps, which are based on gas dynamical calculation. We do not examine processes of transfer of air strike wave energy to soil and structures (parking columns, ceiling, ground, and side walls) and do not contemplate their movements into calculation, i.e. the boundaries of the air space are stiff.

In the first step we calculate the beginning stage of the process of impacts of explosion and spread out of the air strike wave until the moment of contact with the structures.

Calculation of strike wave parameters at the beginning stage is base on numerical calculation of one dimension equations of gas dynamic. Distribution of density $\rho(\boldsymbol{r})$, velocity $\boldsymbol{u}(\boldsymbol{r})$ and internal energy $\varepsilon(\boldsymbol{r})$ ( $\boldsymbol{r}$ is the radius or 
distance from the origin, which is centered at the point of explosion) at the moment of beginning of the interaction of the air strike wave with nearest structures used for calculation of spread out and various interactions among air strike waves are incorporated in the interfacial conditions with the second stage.

In the second step processes of interaction of the air strike waves with structures are studies. Strike wave parameters appearing in the second step are computed from equations of three-dimensional gas dynamic.

\section{Equations of motion, calculated parameters, quantities and their dimensions}

Mathematical modeling of the air movements is base on the solution of equations of gas dynamics, which for three-dimensional problem in Cartesian system of coordinates are listed as:

$$
\begin{gathered}
\frac{\partial \rho}{\partial t}+\frac{\partial\left(\rho u_{x}\right)}{\partial x}+\frac{\partial\left(\rho u_{y}\right)}{\partial y}+\frac{\partial\left(\rho u_{z}\right)}{\partial z}=0 \\
\frac{\partial\left(\rho u_{x}\right)}{\partial t}+\frac{\partial\left(p+\rho u_{x}^{2}\right)}{\partial x}+\frac{\partial\left(\rho u_{x} u_{y}\right)}{\partial y}+\frac{\partial\left(\rho u_{x} u_{z}\right)}{\partial z}=0 \\
\frac{\partial\left(\rho u_{y}\right)}{\partial t}+\frac{\partial\left(\rho u_{x} u_{y}\right)}{\partial x}+\frac{\partial\left(p+\rho u_{y}^{2}\right)}{\partial y}+\frac{\partial\left(\rho u_{y} u_{z}\right)}{\partial z}=0 \\
\frac{\partial\left(\rho u_{z}\right)}{\partial t}+\frac{\partial\left(\rho u_{x} u_{z}\right)}{\partial x}+\frac{\partial\left(\rho u_{y} u_{z}\right)}{\partial y}+\frac{\partial\left(p+\rho u_{z}^{2}\right)}{\partial z}=0 \\
\frac{\partial e}{\partial t}+\frac{\partial\left[(e+p) u_{x}\right]}{\partial x}+\frac{\partial\left[(e+p) u_{y}\right]}{\partial y}+\frac{\partial\left[(e+p) u_{z}\right]}{\partial z}=0
\end{gathered}
$$

where:

$x, y, z$ Cartesian coordinates [m]

$u_{x}, u_{y}, u_{z} \quad$ components of the vectors of velocity $U,[\mathrm{~m} / \mathrm{msec}]$

$|U|^{2}=u_{x}^{2}+u_{y}^{2}+u_{z}^{2} \quad$ norm of the vector of velocity

$\rho=\rho(x, y, z, t) \quad$ density of gas $\left[\mathrm{kg} / \mathrm{m}^{3}\right]$

$p=p(x, y, z, t) \quad$ pressure of gas [MPa]

$e=\rho\left[\varepsilon-\left(u_{x}^{2}+u_{y}^{2}+u_{z}^{2}\right) / 2\right]$ full energy of a unit of mass of the gas, [MPa]

$\varepsilon=\varepsilon(x, y, z, t) \quad$ potential energy $\left[\mathrm{m}^{2} / \mathrm{ms}^{2}\right]$

$\frac{1}{2}\left(u_{x}^{2}+u_{y}^{2}+u_{z}^{2}\right) \quad$ kinetic energy $\left[\mathrm{m}^{2} / \mathrm{ms}^{2}\right]$

Equation describing explosion of TNT charge can be recorded as

$$
p=(\gamma-1) \rho \varepsilon,
$$

where $\gamma$ is the exponent of adiabatic process. For the air $\gamma=1.4$, in case of explosion the exponent of adiabatic process becomes density dependent, i.e. $\gamma=$ $\gamma(\rho)$. Exponent of adiabatic process $\gamma(\rho)$ can be calculated in the following way 


$$
\begin{array}{llll}
- & \gamma=3 & \text { if } & \rho>440 \mathrm{~kg} / \mathrm{m}^{3} ; \\
- & \gamma=1.3 & \text { if } & \rho<50 \mathrm{~kg} / \mathrm{m}^{3} ; \\
\text { - } & \gamma=\gamma(\rho) & \text { if } & 50 \leq \rho \leq 440 \mathrm{~kg} / \mathrm{m}^{3} \text { - linear interpolation }
\end{array}
$$

can be applied (monotonic and smooth dependence on density $\rho$ is assumed).

Using matrix notation,

$$
\boldsymbol{\sigma}=\left\{\begin{array}{c}
\rho \\
\rho u_{x} \\
\rho u_{y} \\
\rho u_{z} \\
e
\end{array}\right\}, \quad \boldsymbol{a}=\left[\begin{array}{c}
\rho u_{x} \\
p+\rho u_{x}^{2} \\
\rho u_{x} u_{y} \\
\rho u_{x} u_{z} \\
(e+p) u_{x}
\end{array}\right], \boldsymbol{b}=\left[\begin{array}{c}
\rho u_{y} \\
\rho u_{x} u_{y} \\
p+\rho u_{y}^{2} \\
\rho u_{y} u_{z} \\
(e+p) u_{y}
\end{array}\right], \boldsymbol{c}=\left[\begin{array}{c}
\rho u_{z} \\
\rho u_{x} u_{z} \\
\rho u_{y} u_{z} \\
p+\rho u_{z}^{2} \\
(e+p) u_{z}
\end{array}\right] .
$$

the above equations can be recorded in a simpler form as $\frac{\partial \sigma}{\partial t}+\frac{\partial a}{\partial x}+\frac{\partial b}{\partial y}+\frac{\partial c}{\partial z}=0$.

If the formulation possesses certain kind of symmetry three dimensional equations can be transformed into one dimensional equations of the form:

$$
\begin{gathered}
\frac{\partial \rho}{\partial t}+\frac{\partial(\rho u)}{\partial r}+\frac{(v-1) \rho u}{r}=0 \\
\frac{\partial(\rho u)}{\partial t}+\frac{\partial\left(p+\rho u^{2}\right)}{\partial r}+\frac{(v-1) \rho u^{2}}{r}=0 \\
\frac{\partial e}{\partial t}+\frac{\partial[(e+p) u]}{\partial r}+\frac{(v-1)[(e+p) u]}{r}=0
\end{gathered}
$$

where $r$ is the space coordinate, $v$ is a sign of symmetry $(v=1$ - plane, 2 cylindrical, 3 - spherical symmetry). In case of cylindrical symmetry axial coordinate $z$ is not considered in the above formulas. The nonlinear equations are solved by the method of Godunov et al. [8] using special linearization of the above equations.

\section{Regularization of functions and their derivatives}

The concept behind SHP is based on an interpolation scheme. From mathematical calculus it is well known, [7], that for each generalized function $f$ defined on a domain $V \subset R^{n}$ with boundary $S$ there exists a positive $\varepsilon$ and a finite cover $\{\Omega\}_{i=1}^{N} \subset V, i=1, \ldots, N$ (for each point $x \in V$ there is an index $i \in 1, \ldots, N$ so that $x \in \Omega_{i}$ ) with measure of $\Omega_{i}<\varepsilon$ so that on $\Omega_{i}$ there exists function $\omega_{\varepsilon}^{i} \in C^{\infty}(\bar{\Omega})$, supp $\omega_{\varepsilon}^{i} \in \Omega_{i}$ (sometimes called cap function) which regularize the function $f$ in such a way that $f$ can be expressed as

$$
f(\boldsymbol{x})=\sum_{i=1}^{N} \int_{\Omega_{i}} f(\boldsymbol{\xi}) \omega_{\varepsilon}^{i}(\boldsymbol{x}-\boldsymbol{\xi}) \mathrm{d} \boldsymbol{\xi}=f^{*} \omega_{\varepsilon}^{i}, i=1, \ldots, N
$$


and the left hand side of the latter relation is called the regularization, $f^{*} \omega_{\varepsilon}^{i}$ is the convolution. Recall some basic properties of the regularization: the volume of each cap function is unity, is equal to one. If the function $f$ is uniform (equal to one) and $\varepsilon \rightarrow$ infinity the regularization turns to be density of the function $f$, for example density of probability. If $\varepsilon \rightarrow 0$ the kernel $\omega_{\varepsilon}^{i}$ turns to be the Dirac function. For each positive $\varepsilon$ the regularization (kernel, cap function) $\omega_{\varepsilon}^{i}$ can be created infinitely differentiable (for definition of types of cap functions, see [7], for example).

Since different cap functions should be created for different $\Omega_{i}$, the above definition becomes inconvenient. In order to improve this put $\omega_{\varepsilon}^{i} \equiv \omega_{\varepsilon}$ and the shape of $\Omega_{i}$ remains same for all $i$, the area of a circle in 2D or the volume of a sphere, for example. Now inside of the domain $V$ select a set of points $\boldsymbol{x}_{i}, i=1, \ldots, N, \boldsymbol{x}_{i}$ is centered at $\Omega_{i}$ and a new function $F$ is defined as

$$
F\left(\boldsymbol{x}_{i}\right)=\sum_{i=1}^{N} \int_{\Omega_{i}} f(\boldsymbol{\xi}) \omega_{\varepsilon}\left(\boldsymbol{x}_{i}-\boldsymbol{\xi}\right) \mathrm{d} \boldsymbol{\xi}, i=1, \ldots, N
$$

which is formally similar to relation (1), so that it fulfils basic properties above mentioned. Since the former assumptions take place the function $F$ cannot be expected to be equal to $f$ any longer, but a special case: $\varepsilon \rightarrow 0$ in the sense of definition of the Dirac function.

In our case $2 \mathrm{D}$ problem is considered and degrees of freedom are concentrated at nodes $\boldsymbol{x}_{i} \in \Omega_{i}, i=1, \ldots, N, \Omega_{i}$ are considered as areas of the circles in which $x_{i}$ is centered. In the approximation, the smoothed (regularized) function $F$ for any physical quantity $f$ is identified with the original function, i.e. $F \equiv f$. Moreover, the kernel $\omega_{\varepsilon}$ is simplifies for real calculations and the simplification is denoted as $W_{\varepsilon}$. Introducing this to (2) and setting $f_{i}=f\left(\boldsymbol{x}_{i}\right)$ gives:

$$
f_{i}=f\left(\boldsymbol{x}_{i}\right)=\int_{\Omega_{i}} f(\boldsymbol{\xi}) W_{\varepsilon}\left(\boldsymbol{x}_{i}-\boldsymbol{\xi}\right) \mathrm{d} \boldsymbol{\xi}
$$

Equation (3) is the kernel representation to average functional distribution. In our next considerations additional properties of $W_{\varepsilon}$ will be required:

$$
\begin{array}{cr}
\text { positivity: } & W_{\varepsilon}\left(\boldsymbol{x}_{i}-\boldsymbol{\xi}\right) \geq 0, \quad \boldsymbol{\xi} \in \Omega_{i} \\
\text { normalization } & \int_{\Omega_{i}} W_{\varepsilon}\left(\boldsymbol{x}_{i}-\boldsymbol{\xi}\right) \mathrm{d} \boldsymbol{\xi}=1, \quad \forall \varepsilon>0 \\
\text { surface smoothness on } \partial \Omega_{i}: \begin{array}{r}
W_{\varepsilon}\left(\boldsymbol{x}_{i}-\boldsymbol{\xi}\right)=\nabla W_{\varepsilon}\left(\boldsymbol{x}_{i}-\boldsymbol{\xi}\right)= \\
=\nabla \nabla W_{\varepsilon}\left(\boldsymbol{x}_{i}-\boldsymbol{\xi}\right)=0, \quad \boldsymbol{\xi} \in \partial \Omega_{i}
\end{array}
\end{array}
$$

The last property follows from the fact that the order of differential equations, which are to be studied, is two, and so is the required regularity (continuity). 
Using integration by parts, from the boundary conditions on $\partial \Omega_{i}$ it immediately follows that

$$
\int_{\Omega} \nabla W_{\varepsilon}\left(\boldsymbol{x}_{i}-\boldsymbol{\xi}\right) \mathrm{d} \boldsymbol{\xi}=\int_{\Omega} \nabla \nabla W_{\varepsilon}\left(\boldsymbol{x}_{i}-\boldsymbol{\xi}\right) \mathrm{d} \boldsymbol{\xi}=0
$$

For the sake of simplicity the approximation of the kernel $W_{\varepsilon}$ is represented by

$$
W_{\varepsilon}\left(\boldsymbol{x}_{i}-\boldsymbol{\xi}\right)=C\left(1-10 r^{3}+15 r^{4}-6 r^{5}\right), \quad r=\left|\boldsymbol{\xi}-\boldsymbol{x}_{i}\right| / h \quad \text { for } 2 \mathrm{D}
$$

where $C=\frac{1}{h}$ for $1 \mathrm{D}$ problem, $C=\frac{7}{3 \pi h^{2}}$ for $2 \mathrm{D}$ problem, $C=\frac{42}{5 \pi h^{3}}$ for $3 \mathrm{D}$ problem, and $\left|\boldsymbol{\xi}-\boldsymbol{x}_{i}\right| \leq h$ is the distance between the pertinent points.

If we consider volume (area, interval) of an element $\Omega_{i}=\frac{m_{i}}{\rho_{i}}$, where $m_{i}$ is the mass of the element and $\rho_{i}$ is the density, using rectangular rule of evaluation of integrals yields:

$$
\begin{gathered}
f_{i}=f\left(x_{i}\right)=\sum_{r_{i j} \leq h} \frac{m_{j} f_{j}}{\rho_{j}} W_{\varepsilon}\left(r_{i j}\right), r_{i j}=\left|\boldsymbol{x}_{j}-\boldsymbol{x}_{i}\right|, \\
\nabla f_{i}=\rho_{i} \sum_{r_{i j} \leq h} m_{j}\left(\frac{f_{i}}{\rho_{i}^{2}}+\frac{f_{j}}{\rho_{j}^{2}}\right) \nabla W_{\varepsilon}\left(r_{i j}\right)
\end{gathered}
$$

\section{Calculation schemes}

Typical explosive scheme of three dimensional problem is considered in this section. It starts with the position of the charge near the neighboring side walls. This case is depicted in Figs. 1, where in the left picture view of the situation and in the right picture the plot of the situation is seen. The ball centered at the charge position with radius $R_{\mathrm{c}}$ describes the domains of charge in the picture.

The charge position as well as the side walls, ground and ceiling are imbedded in Cartesian coordinates $0 x y z$, where the plane $0 x y$ is the ground and $z$ is upwards oriented. In both cases the ground is characterized by the plane $z=0$ and the ceiling is the plane $z=3$. The length dimensions are measured in meters. Center of the charge possesses the coordinates $\left(0.5+R_{\mathrm{c}}, 0.5+R_{\mathrm{c}}, 0.5+R_{\mathrm{c}}\right)$ in the first case and in the second case vertical coordinate is $0.5+R_{\mathrm{c}}$, while the other coordinates are zero. Values of the radii of charge and its mass $q$ are introduced in Table 1 for the density of TNT $\rho_{\mathrm{TNT}}=1620 \mathrm{~kg} / \mathrm{m}^{3}$.

In the first stage of definition of loads numerical solution of the problem with the air explosion charges of mass $q=50 \mathrm{~kg}$ and $q=100 \mathrm{~kg}$ TNT till the front $r_{0}=$ $0.5+R_{\mathrm{c}}$.

Characterization of the charge is done by definition of mass and energy inside of the domain of charge. Density of TNT is considered as $1620 \mathrm{~kg} / \mathrm{m}^{3}$. 

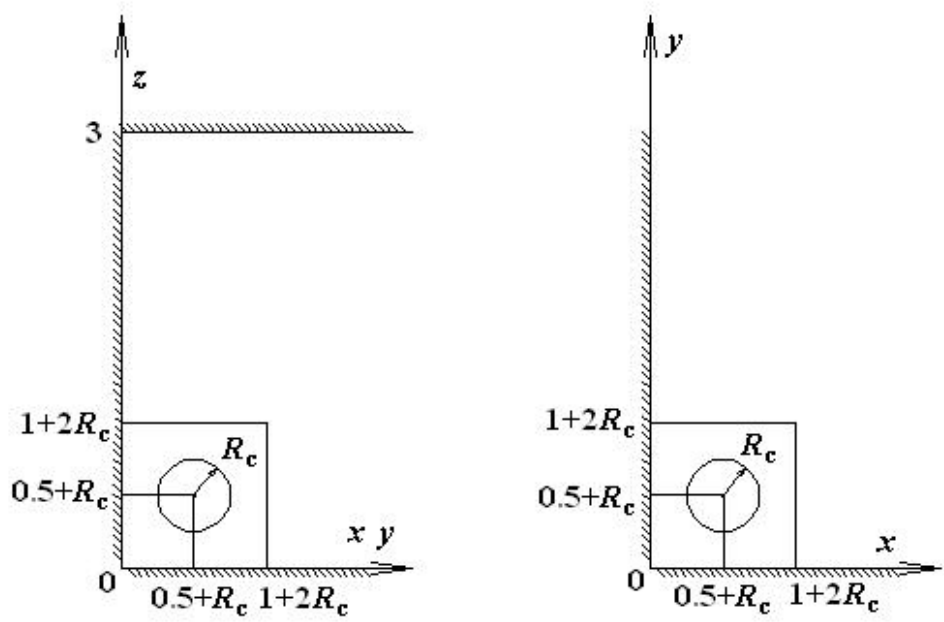

Figure 1: $\quad$ Scheme for the charge position near the sidewalls.

Table 1: $\quad$ Dependency of the radius $R_{\mathrm{c}}$ on the power $q$.

\begin{tabular}{|c|c|}
\hline$q, \mathrm{~kg}$ & $R_{\mathrm{c}}, \mathrm{m}$ \\
\hline 50 & 0.245 \\
\hline 100 & 0.195 \\
\hline
\end{tabular}

Table 2: $\quad$ Remaining pressure $\Delta p_{f}$.

\begin{tabular}{|c|c|c|}
\hline$q, \mathrm{~kg}$ & $R_{f}, \mathrm{~m}$ & $\Delta p_{f}, \mathrm{kgs} / \mathrm{cm}^{2}$ \\
\hline 50 & 0.695 & 131 \\
\hline 100 & 0.745 & 156.7 \\
\hline
\end{tabular}

\section{Results}

Results of calculation - beginning distribution of density $\rho(r)$, speed $u(r)$ and pressure $p(r)$ behind the air strike wave are shown in Figs. 2-7 for the case depicted in Fig. 1. In the graphs of density distribution $\rho(r)$ drop of this function behind the air strike wave is seen. Density disconnection is equal to contact drop that divides influences of charge and the air compressed by the air strike wave. The boundary conditions on the interface of the air and the structures of the parking are prescribed in such a way that fully reflexive surfaces of the structures are considered.

In Table 2 the remaining pressure $\Delta p_{f}$ on the front of air strike wave at the beginning of interaction of the air strike wave and the structure for the first case of geometry, see Fig. 1. 


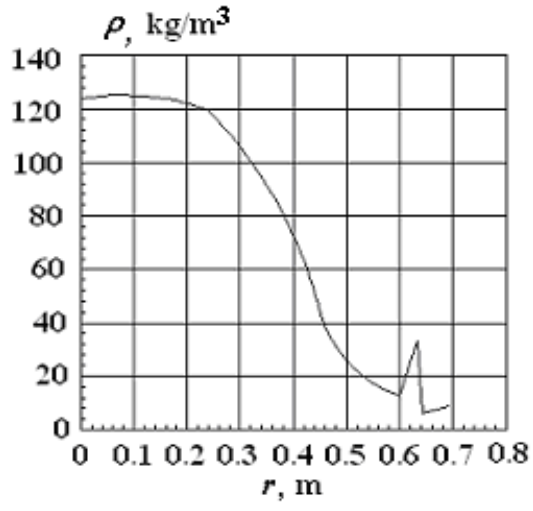

Figure 2: $\quad$ Initial density for $q=$ 50 .

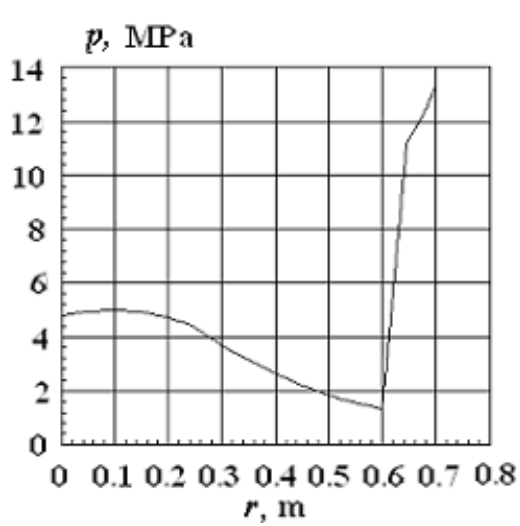

Figure 4: $\quad$ Initial pressure for $q$ $=50$.

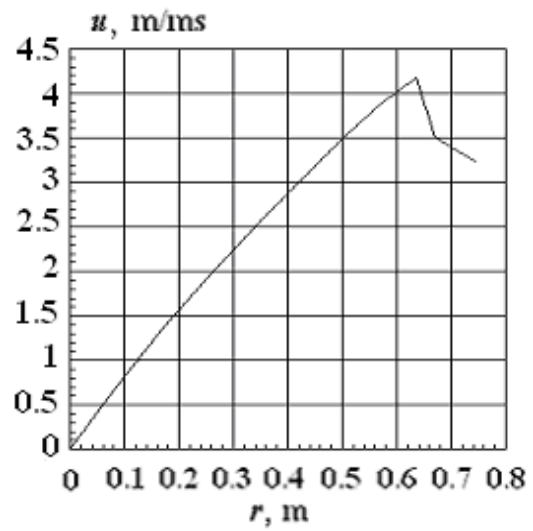

Figure 6: Initial velocity for $q=100$.

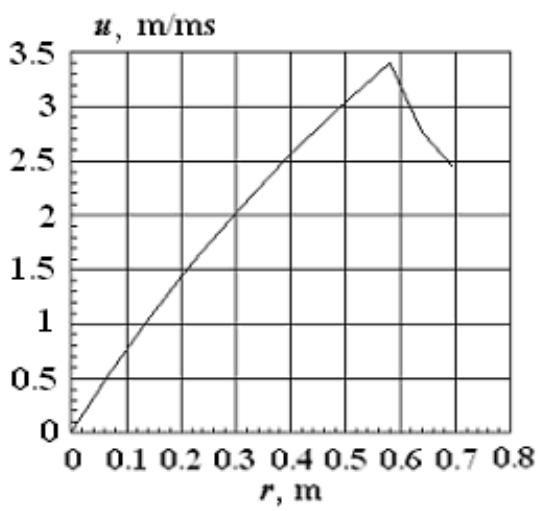

Figure 3: $\quad$ Initial velocity for $q$ $=50$.

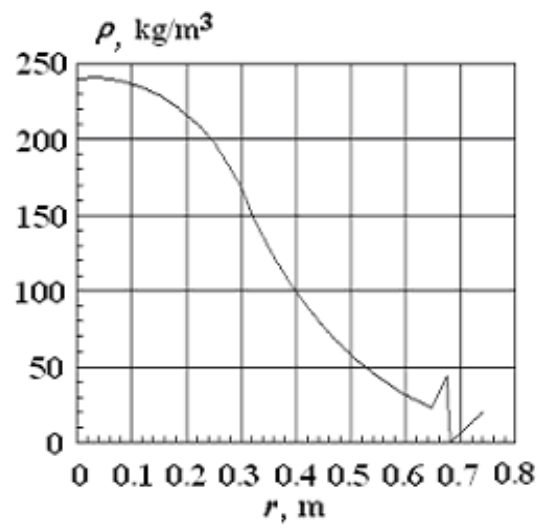

Figure 5: $\quad$ Initial density for $q=$ 100.

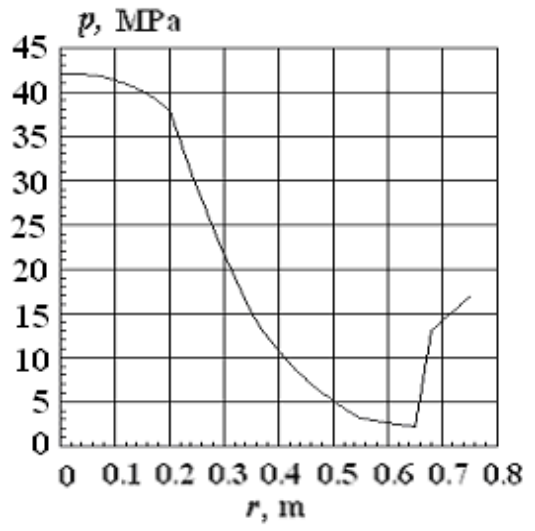

Figure 7: Initial pressure for $q=100$. 


\section{Conclusions}

In this paper movements of gas due to explosion in an underground parking are studied. The main purpose of this paper is to discover the loading developed against the side walls, ground and ceiling of the parking room. The SHP method is used as a numerical tool, solving the set of equations describing the movement process of gas (air). Density disconnection appears at the walls due to difference in influences of the charge and the air, which is compressed by the air strike wave. In the neighborhood of the charge supersonic velocity is considered, which induces subsonic velocity in the air.

\section{Acknowledgements}

This paper was prepared under financial support of GAČR, project No. 103/08/0922 and MSM, project number 6840770001.

\section{References}

[1] Lucy, L.B. (1977). A numerical approach to testing of the fission hypothesis. Astron. J. 82, 1013.

[2] Gingold, R.A. and Monoghan, J.J. (1977). Smooth particle hydrodynamics: theory and application to non-spherical stars. Monthly Nat. R. Astron. Soc. 181, 375.

[3] Randle, P.W. and Libersky, L.D. (1996). Smooth particle hydrodynamics: some recent improvements and application. Appl. Mech. Engng. 139, 175.

[4] Li, S. and Liu, W.K. (2002). Meshfree and particle method and their applications. Appl. Mech. Rev. 55, 1.

[5] Takeda, H., Miyama, S. and Sekiya, M. (1994). Numerical simulation of viscous flow by smoothed particle hydrodynamics. Prog. Theor. Phys. 92, 939.

[6] Chen, J.K., Beraun, J.E. and Carney, T.C. (1999). A corrective smooth particle method for boundary value problems in heat conduction. Int. J. Numer. Methods Engrg. 46, 231.

[7] Veselovsky, A.N., Kurepin, N.S.: Proceedings of $26^{\text {th }}$ Central Scientific Institute of the Russian Federation. Moscow 2006, report II/2, in Russian

[8] Godunov, S.K., Zabrodin, A.V. et.al: Numerical solutions of the polydimensional problem of gas dynamic. Moscow, Nauka, 1976, in Russian.

[9] Zeldovich, J.B., Raize, J.P.: Physics of strike waves and high temperature hydrodynamic processes. Moscow, Nauka, 1966, in Russian. 